\title{
Commentary to the Article "On Calculation of the Transport Coefficients and Thermodynamic Properties of a Helium-Xenon Gas Mixture" by Yu. G. Dragunov, V. P. Smetannikov, B. A. Gabaraev, A. N. Orlov, M. S. Belyakov, and D. S. Derbenev
}

\author{
V. E. Nakoryakov \\ Kutateladze Institute of Thermophysics, Siberian Branch, Russian Academy of Sciences, \\ pr. Akad. Lavrent'eva 1, Novosibirsk, 630090 Russia \\ Received February 23, 2012
}

DOI: $10.1134 / \mathrm{S} 1810232813010037$

The authors of the article have considered the issue and presented a detailed review of investigation on the heat transfer of light and heavy noble gas flows in channels, using xenon and helium as an example. The heat transfer in these mixtures has been insufficiently experimentally studied because xenon is hardly available to researchers due to its high price. The interest in using these mixtures for theoretical thermophysics is tremendous. The great difference in masses of xenon and helium leads to the necessity of more thorough study. From my point of view, initial works for analysis can be those on investigation of helium, which has been profoundly studied by Tisza, Kapitza, and Landau. For explaining the effects of superfluidity and superconductivity, they had to introduce two-velocity consideration on a purely hydrodynamic base, they introduced a drift velocity, which had made it possible to explain both super thermal conductivity and extinct viscosity effects. In the case of fluids with a profoundly different density, there always appear effects that cannot be explained from the initial viewpoint. For example, sound velocity in a fluid with gas bubbles.

While investigating a xenon-helium mixture, some mysterious phenomena have been found, namely, the Prandtl number of the mixture differs from Prandtl numbers of xenon and helium, taken separately. From the viewpoint of molecular physics, this fact is more or less clear when introducing a drift velocity between two molecules. Moreover, it is clear that for two molecules with different path lengths, there inevitably occurs interdiffusion if a temperature gradient is present. To verify this fact, it necessary to perform a series of investigations as well as some additional investigations in a turbulent flow in the zone of a laminar sublayer. In this case, one has to consider not only the thermal conductivity equation, but also the diffusion equation.

In these mixtures, such effects of decreasing sound velocity are possible, that is, the sound velocity in the mixture can be lower than that in each of the phases. This guess has immediately appeared in my mind while inspecting the problem because derivation of velocity only for a liquid-gas mixture does not formally differ from derivation of sound velocity in a mixture of heavy and light gas. The most interesting point is that after having a look at Table 2.18 (Sound velocity of helium-xenon mixture with a mass fraction of He $7.17 \%$ ) in [32] and comparing separately to the sound velocity for He and Xe, we see that my guess is true. It is necessary to investigate acoustic processes in these mixtures. Probably, is some parts of the package, one has to consider compressibility of the mixture. 